\title{
STABILITY OF GORENSTEIN FLAT CATEGORIES WITH RESPECT TO A SEMIDUALIZING MODULE
}

\author{
ZHENXING DI, ZHONGKUI LIU AND JIANLONG CHEN
}

ABSTRACT. We first introduce in the paper the $\mathcal{W}_{F^{-}}$ Gorenstein modules to establish the following Foxby equivalence:

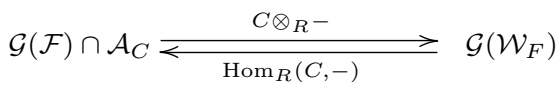

where $\mathcal{G}(\mathcal{F}), \mathcal{A}_{C}$ and $\mathcal{G}\left(\mathcal{W}_{F}\right)$ denote the class of Gorenstein flat modules, the Auslander class and the class of $\mathcal{W}_{F^{-}}$ Gorenstein modules, respectively. Then, we investigate twodegree $\mathcal{W}_{F}$-Gorenstein modules. An $R$-module $M$ is said to be two-degree $\mathcal{W}_{F}$-Gorenstein if there exists an exact sequence $\mathbb{G}_{\bullet}=\cdots \rightarrow G_{1} \rightarrow G_{0} \rightarrow G^{0} \rightarrow G^{1} \rightarrow \cdots$ in $\mathcal{G}\left(\mathcal{W}_{F}\right)$ such that $M \cong \operatorname{im}\left(G_{0} \rightarrow G^{0}\right)$ and $\mathbb{G}_{\bullet}$ is $\operatorname{Hom}_{R}\left(\mathcal{G}\left(\mathcal{W}_{F}\right),-\right)$ and $\mathcal{G}\left(\mathcal{W}_{F}\right)^{+} \otimes_{R}-$ exact. We show that two notions of the two-degree $\mathcal{W}_{F}$-Gorenstein and the $\mathcal{W}_{F}$-Gorenstein modules coincide when $R$ is a commutative $G F$-closed ring.

1. Introduction. Throughout this article, $R$ is a commutative ring with identity and all modules are unitary. We denote by $R$-Mod the category of $R$-modules. For an $R$-module $M$, the Pontryagin dual or character module $\operatorname{Hom}_{\mathbb{Z}}(M, \mathbb{Q} / \mathbb{Z})$ is denoted by $M^{+}$.

Recall from White [11] that an $R$-module $C$ is said to be semidualizing if $C$ admits a degreewise finite projective resolution, the natural homothety map $R \rightarrow \operatorname{Hom}_{R}(C, C)$ is an isomorphism and $\operatorname{Ext}_{R}^{\geqslant 1}(C, C)=0$. Examples include the rank one free modules and a dualizing (canonical) module when one exists. With this notion, the Auslander class and the Bass class with respect to a fixed semidualizing $R$-module $C$, denoted by $\mathcal{A}_{C}$ and $\mathcal{B}_{C}$, respectively, can be defined

2010 AMS Mathematics subject classification. Primary 16E05, 16E10, 55 U15.

Keywords and phrases. Semidualizing module, $G_{C}$-flat module, $\mathcal{W}_{F}$-Gorenstein module, Bass class, stability of category.

This work is supported by the National Natural Science Foundation of China (Nos. 11261050 and 11371089), and the Specialized Research Fund for the Doctoral Program of Higher Education (No. 20120092110020).

Received by the editors on September 19, 2012, and in revised form on January 16, 2014. 
and studied naturally. It is well known that there exists the following equivalence of categories:

$$
\mathcal{A}_{C} \underset{\operatorname{Hom}_{R}(C,-)}{\rightleftarrows} \mathcal{B}_{C} .
$$

Recently, as a generalization of the classes of Gorenstein projective and Gorenstein injective modules, denoted by $\mathcal{G}(\mathcal{P})$ and $\mathcal{G}(\mathcal{I})$, respectively, Geng and Ding [4] introduced the notions of the $\mathcal{W}_{P}$-Gorenstein and the $\mathcal{W}_{I}$-Gorenstein modules. In particular, they obtained the following interesting equivalences of categories:

$$
\mathcal{G}(\mathcal{P}) \cap \mathcal{A}_{C} \underset{\operatorname{Hom}_{R}(C,-)}{\stackrel{C \otimes_{R}-}{\rightleftarrows}} \mathcal{G}\left(\mathcal{W}_{P}\right)
$$

and

$$
\mathcal{G}\left(\mathcal{W}_{I}\right) \underset{\operatorname{Hom}_{R}(C,-)}{\stackrel{C \otimes_{R}-}{\rightleftarrows}} \mathcal{G}(\mathcal{I}) \cap \mathcal{B}_{C},
$$

where $\mathcal{G}\left(\mathcal{W}_{P}\right)$ and $\mathcal{G}\left(\mathcal{W}_{I}\right)$ denote the classes of $\mathcal{W}_{P}$-Gorenstein and $\mathcal{W}_{I^{-}}$ Gorenstein modules, respectively. So it is natural to ask if there exist some other classes satisfying the following diagram:

$$
\mathcal{G}(\mathcal{F}) \cap \mathcal{A}_{C} \underset{\operatorname{Hom}_{R}(C,-)}{\stackrel{C \otimes_{R}-}{\rightleftarrows}} \text { ? }
$$

The motivation of the present article is the "?."

We shall introduce in Section 3 the notion of the $\mathcal{W}_{F}$-Gorenstein module, which plays the role of "?." Combined with $\mathcal{W}_{P}$-Gorenstein and $\mathcal{W}_{I}$-Gorenstein modules, they can be treated from a similar aspect as the relationship among projective, injective and flat modules in classical homological algebra theory. An $R$-module $M$ is said to be $\mathcal{W}_{F}$-Gorenstein if there exists an exact sequence

$$
\mathbb{W}_{\bullet}=\cdots \longrightarrow W_{1} \longrightarrow W_{0} \longrightarrow W^{0} \longrightarrow W^{1} \longrightarrow \cdots
$$

in $\mathcal{F}_{C}$ such that $M \cong \operatorname{im}\left(W_{0} \rightarrow W^{0}\right)$ and $\mathbb{W} \bullet$ is $\operatorname{Hom}_{R}\left(\mathcal{P}_{C},-\right)$ and $\mathcal{I}_{C} \otimes_{R}$ - exact, where $\mathcal{F}_{C}, \mathcal{P}_{C}$ and $\mathcal{I}_{C}$ denote the classes of $C$-flat, $C$-projective and $C$-injective modules, respectively. In particular, we get the following theorem demonstrating the relationship between the classes $\mathcal{G}\left(\mathcal{W}_{F}\right)$ and $\mathcal{G} \mathcal{F}_{C}$ (see Theorem 3.4): 
Theorem I. Let $C$ be a semidualizing $R$-module. Then $\mathcal{G}\left(\mathcal{W}_{F}\right)=$ $\mathcal{G F}_{C} \cap \mathcal{B}_{C}$.

Also, the $\mathcal{G}\left(\mathcal{W}_{F}\right)$-projective dimension for any $R$-module will be discussed in this section.

In Section 4, we first introduce the modules that arise from an iteration of the above construction. To wit, let $\mathcal{G}^{2}\left(\mathcal{W}_{F}\right)$ denote the class of $R$-module $M$ for which there exists an exact sequence

$$
\mathbb{G}_{\bullet}=\cdots \longrightarrow G_{1} \longrightarrow G_{0} \longrightarrow G^{0} \longrightarrow G^{1} \longrightarrow \cdots
$$

in $\mathcal{G}\left(\mathcal{W}_{F}\right)$ such that $M \cong \operatorname{im}\left(G_{0} \rightarrow G^{0}\right)$ and $\mathbb{G}_{\bullet}$ is $\operatorname{Hom}_{R}\left(\mathcal{G}\left(\mathcal{W}_{F}\right),-\right)$ and $\mathcal{G}\left(\mathcal{W}_{F}\right)^{+} \otimes_{R^{-}}$-exact. Similarly, one can also define $R$-modules which belong to $\mathcal{G}^{2}\left(\mathcal{G F}_{C} \cap \mathcal{B}_{C}\right)$ or $\mathcal{G}^{2}(\mathcal{F})$, although the definition above differs from that in [9], there is still a good correspondence. We then apply techniques obtained in the former section to get our results concerning stability properties of Gorenstein categories (see Theorem 4.5, Corollary 4.6 and Corollary 4.7).

Theorem II. Let $R$ be a GF-closed ring and $C$ a semidualizing $R$-module. Then the following hold:

(i) $\mathcal{G}^{2}\left(\mathcal{W}_{F}\right)=\mathcal{G}\left(\mathcal{W}_{F}\right)$.

(ii) $\mathcal{G}^{2}\left(\mathcal{G F}_{C} \cap \mathcal{B}_{C}\right)=\mathcal{G F}_{C} \cap \mathcal{B}_{C}$.

(iii) $\mathcal{G}^{2}(\mathcal{F})=\mathcal{G}(\mathcal{F})$.

In the remainder of the paper, let $C$ be a fixed semidualizing $R$ module. We mainly recall some necessary notions and definitions in the next section.

2. Preliminaries. Let $\mathcal{X}$ and $\mathcal{Y}$ be two classes of $R$-modules. We begin with the following definition.

Definition 2.1. We write $\mathcal{X} \perp \mathcal{Y}$ (respectively, $\mathcal{X} \top \mathcal{Y}$ ) in case $\operatorname{Ext}_{R}^{\geqslant 1}(X, Y)=$ 0 (respectively, $\operatorname{Tor}_{\geqslant 1}^{R}(X, Y)=0$ ) for each object $X \in \mathcal{X}$ and object $Y \in \mathcal{Y}$. For an $R$-module $M$, when $\mathcal{X}=\{M\}$, we use the notation $M \perp \mathcal{Y}$ instead of $\{M\} \perp \mathcal{Y}$. There are some analogues such as $M \top \mathcal{Y}$, $\mathcal{X} \perp M$ and $\mathcal{X} \top M$. Following [10], we say that $\mathcal{X}$ is a generator for $\mathcal{Y}$ if $\mathcal{X} \subseteq \mathcal{Y}$ and for each object $Y \in \mathcal{Y}$, there exists a short exact sequence

$$
0 \longrightarrow Y^{\prime} \longrightarrow X \longrightarrow Y \longrightarrow 0
$$


in $\mathcal{Y}$ such that $X \in \mathcal{X}$. The class $\mathcal{X}$ is a projective generator for $\mathcal{Y}$ if $\mathcal{X}$ is a generator for $\mathcal{Y}$ and $\mathcal{X} \perp \mathcal{Y}$.

Definition 2.2. For any $R$-module $M$, we recall three types of resolutions.

(i) $[5,1.5]$. A left $\mathcal{X}$-resolution of $M$ is an exact sequence $\mathbb{X}=\cdots \rightarrow$ $X_{1} \rightarrow X_{0} \rightarrow M \rightarrow 0$ with $X_{n} \in \mathcal{X}$ for all $n \geqslant 0$.

(ii) $[5,1.5]$. A right $\mathcal{X}$-resolution of $M$ is an exact sequence $\mathbb{X}=0 \rightarrow$ $M \rightarrow X^{0} \rightarrow X^{1} \rightarrow \cdots$ with $X^{n} \in \mathcal{X}$ for all $n \geqslant 0$.

Now let $\mathbb{X}$ be any (left or right) $\mathcal{X}$-resolution of $M$. We say that $\mathbb{X}$ is co-proper if the sequence $\operatorname{Hom}_{R}(\mathbb{X}, X)$ is exact for each object $X \in \mathcal{X}$.

(iii) $[\mathbf{1 1}, 1.6]$. A degreewise finite projective (respectively, free) resolution of $M$ is a left projective (respectively, free) resolution $\mathbb{P}$ of $M$ such that each $P_{i}$ is finitely generated projective (respectively, free). It is easy to verify that $M$ admits a degreewise finite projective resolution if and only if $M$ admits a degreewise finite free resolution.

Definition 2.3. The $\mathcal{X}$-projective dimension of an $R$-module $M$ is defined as:

$$
\begin{aligned}
& \mathcal{X}-\operatorname{pd}_{R}(M) \\
& \quad=\inf \left\{\sup \left\{n \mid X_{n} \neq 0\right\} \mid \mathbb{X} \text { is a left } \mathcal{X} \text {-resolution of } M\right\} .
\end{aligned}
$$

Dually, one can also define the $\mathcal{X}$-injective dimension of $M$.

The next lemma has a standard proof.

Lemma 2.4. Let $M$ be an $R$-module. Consider the following exact sequence in $\mathcal{X}$ :

$$
\mathbb{X}=\cdots \longrightarrow X_{1} \stackrel{\delta_{1}^{\mathbb{X}}}{\longrightarrow} X_{0} \stackrel{\delta_{0}^{\mathbb{X}}}{\longrightarrow} X_{-1} \longrightarrow \cdots .
$$

Then the following hold:

(i) Assume $M \perp \mathcal{X}$. If $\mathbb{X}$ is $\operatorname{Hom}_{R}(M,-)$ exact, then $\operatorname{Ext}_{R}^{\geqslant 1}\left(M, \operatorname{im}\left(\delta_{i}^{\mathbb{X}}\right)\right)$ $=0$ for all $i$. Conversely, if $\operatorname{Ext}_{R}^{1}\left(M, \operatorname{im}\left(\delta_{i}^{\mathbb{X}}\right)\right)=0$ for all $i$, then $\mathbb{X}$ is $\operatorname{Hom}_{R}(M,-)$ exact. 
(ii) Assume $M \top \mathcal{X}$. If $\mathbb{X}$ is $M \otimes_{R}$-exact, then $\operatorname{Tor}_{\geqslant 1}^{R}\left(M, \operatorname{im}\left(\delta_{i}^{\mathbb{X}}\right)\right)=0$ for all $i$. Conversely, if $\operatorname{Tor}_{1}^{R}\left(M, \operatorname{im}\left(\delta_{i}^{\mathbb{X}}\right)\right)=0$ for all $i$, then $\mathbb{X}$ is $M \otimes_{R}$-exact.

Definition 2.5. [3]. An $R$-module $M$ is said to be Gorenstein flat if there exists an exact sequence

$$
\mathbb{X}=\cdots \longrightarrow F_{1} \longrightarrow F_{0} \longrightarrow F^{0} \longrightarrow F^{1} \longrightarrow \cdots
$$

in $R$-Mod with each $F_{i}$ and $F^{i}$ flat such that $M \cong \operatorname{im}\left(F_{0} \rightarrow F^{0}\right)$ and $\mathbb{X}$ is $I \otimes_{R}$-exact for any injective $R$-module $I$. The exact sequence $\mathbb{X}$ is called complete flat resolution of $M$.

In the following, we denote the class of Gorenstein flat modules by $\mathcal{G}(\mathcal{F})$.

Definition 2.6. [1]. Let $R$ be a ring. We call $R$ GF-closed if the class of Gorenstein flat $R$-modules is closed under extensions, that is, if $0 \rightarrow X \rightarrow Y \rightarrow Z \rightarrow 0$ is a short exact sequence with $X$ and $Z$ Gorenstein flat modules, then $Y$ is also Gorenstein flat.

It follows from [1] that the class of GF-closed rings includes strictly the one of coherent rings and the one of rings of finite weak global dimension.

Definition 2.7. [7]. An $R$-module is $C$-projective (respectively, $C$ flat) if it has the form $C \otimes_{R} P$ for some projective (respectively, flat) $R$-module $P$. An $R$-module is $C$-injective if it has the form $\operatorname{Hom}_{R}(C, I)$ for some injective $R$-module $I$. We set:

$$
\begin{aligned}
& \mathcal{P}_{C}=\left\{C \otimes_{R} P \mid P \text { is a projective } R \text {-module }\right\} \\
& \mathcal{F}_{C}=\left\{C \otimes_{R} F \mid F \text { is a flat } R \text {-module }\right\} \\
& \mathcal{I}_{C}=\left\{\operatorname{Hom}_{R}(C, I) \mid I \text { is an injective } R \text {-module }\right\} .
\end{aligned}
$$

Remark 2.8. The classes defined above are studied extensively in [7]. From [7], we know that 
(i) The classes $\mathcal{F}_{C}$ and $\mathcal{P}_{C}$ are closed under arbitrary direct sums and summands, and, if $R$ is coherent, then $\mathcal{F}_{C}$ is also closed under arbitrary direct products.

(ii) The class $\mathcal{I}_{C}$ is closed under arbitrary direct products and summands.

Definition 2.9. [6]. An $R$-module $N$ is said to be $G_{C}$-injective $\left(G_{C^{-}}\right.$ inj for short) if there exists an exact sequence

$$
\mathbb{Y}=\cdots \longrightarrow \operatorname{Hom}_{R}\left(C, I^{1}\right) \longrightarrow \operatorname{Hom}_{R}\left(C, I^{0}\right) \longrightarrow I_{0} \longrightarrow I_{1} \longrightarrow \cdots
$$

in $R$-Mod with each $I_{i}$ and $I^{i}$ injective such that $N \cong \operatorname{im}\left(\operatorname{Hom}_{R}\left(C, I^{0}\right) \rightarrow\right.$ $\left.I_{0}\right)$ and $\mathbb{Y}$ is $\operatorname{Hom}_{R}\left(\mathcal{I}_{C},-\right)$ exact. The exact sequence $\mathbb{Y}$ is called a complete $\mathcal{I}_{C} \mathcal{I}$-resolution of $N$.

An $R$-module $T$ is said to be $G_{C}$-flat if there exists an exact sequence

$$
\mathbb{Z}=\cdots \longrightarrow F_{1} \longrightarrow F_{0} \longrightarrow C \otimes_{R} F^{0} \longrightarrow C \otimes_{R} F^{1} \longrightarrow \cdots
$$

in $R$-Mod with each $F_{i}$ and $F^{i}$ flat such that $M \cong \operatorname{im}\left(F_{0} \rightarrow C \otimes_{R} F^{0}\right)$ and $\mathbb{Z}$ is $\mathcal{I}_{C} \otimes_{R}-$ exact. The exact sequence $\mathbb{Z}$ is called a complete $\mathcal{F} \mathcal{F}_{C}$-resolution of $T$.

We will denote the classes of $G_{C}$-inj and $G_{C}$-flat modules by $\mathcal{G I}_{C}$ and $\mathcal{G F}_{C}$, respectively.

Remark 2.10. Similar to the proofs in [11] one can easily get that:

(i) Every $C$-injective $R$-module is $G_{C}$-inj, and the class $\mathcal{G I}_{C}$ is coresolving and closed under arbitrary direct products and summands.

(ii) Every $C$-flat $R$-module is $G_{C}$-flat, and the class $\mathcal{G F}_{C}$ is closed under arbitrary direct sums.

(iii) Every kernel of a complete $\mathcal{I}_{C} \mathcal{I}$-resolution (respectively, $\mathcal{F} \mathcal{F}_{C^{-}}$ resolution) belongs to $\mathcal{G I}_{C}$ (respectively, $\mathcal{G F}_{C}$ ).

By using the definition of $G_{C}$-flat modules and Remark 2.10, the proof of the next lemma is a standard argument.

Lemma 2.11. The following are equivalent for an $R$-module $M$ :

(i) $M$ is $G_{C}$-flat.

(ii) $M$ satisfies the following two conditions: 
(a) $\mathcal{I}_{C} \top M$ and

(b) There exists an exact sequence $0 \rightarrow M \rightarrow C \otimes_{R} F^{0} \rightarrow$ $C \otimes_{R} F^{1} \rightarrow \cdots$ in $R$-Mod with each $F^{i}$ flat such that $\mathcal{I}_{C} \otimes_{R}-$ leaves it exact.

(iii) There exists a short exact sequence $0 \rightarrow M \rightarrow C \otimes_{R} F \rightarrow G \rightarrow 0$ in $R$-Mod with $F$ flat and $G \in \mathcal{G F}_{C}$.

Definition $2.12([7])$. The Auslander class $\mathcal{A}_{C}$ with respect to $C$ consists of all $R$-modules $M$ satisfying:

(i) $\operatorname{Tor}_{\geqslant 1}^{R}(C, M)=0=\operatorname{Ext}_{R}^{\geqslant 1}\left(C, C \otimes_{R} M\right)$ and

(ii) The natural evaluation map $\mu_{C C M}: M \rightarrow \operatorname{Hom}_{R}\left(C, C \otimes_{R} M\right)$ is an isomorphism.

Dually, the Bass class $\mathcal{B}_{C}$ with respect to $C$ consists of all $R$ modules $N$ satisfying

(a) $\operatorname{Ext}_{R}^{\geqslant 1}(C, N)=0=\operatorname{Tor}_{\geqslant 1}^{R}\left(C, \operatorname{Hom}_{R}(C, N)\right)$, and

(b) The natural evaluation map $\nu_{C C N}: C \otimes_{R} \operatorname{Hom}_{R}(C, N) \rightarrow N$ is an isomorphism.

We now display some necessary results about the classes $\mathcal{A}_{C}$ and $\mathcal{B}_{C}$.

Lemma 2.13. ([7]). The following hold:

(i) If any two $R$-modules in a short exact sequence are in $\mathcal{A}_{C}$, respectively $\mathcal{B}_{C}$, then so is the third.

(ii) The class $\mathcal{A}_{C}$ contains all modules of finite flat dimension and those of finite $\mathcal{I}_{C}$-injective dimension. The class $\mathcal{B}_{C}$ contains all modules of finite injective dimension and those of finite $\mathcal{F}_{C}-$ projective dimension.

To be a direct corollary of [7, Theorem 6.4], we have the following lemma:

Lemma 2.14. $\mathcal{P}_{C} \perp, \mathcal{B}_{C}, \mathcal{A}_{C} \perp \mathcal{I}_{C}$ and $\mathcal{A}_{C} \top \mathcal{F}_{C}$.

3. $\mathcal{W}_{F}$-Gorenstein modules. We begin this section with the following notion of a $\mathcal{W}_{F}$-Gorenstein module. 
Definition 3.1. An $R$-module $M$ is said to be $\mathcal{W}_{F}$-Gorenstein if there exists an exact sequence

$$
\mathbb{W}_{\bullet}=\cdots \longrightarrow W_{1} \longrightarrow W_{0} \longrightarrow W^{0} \longrightarrow W^{1} \longrightarrow \cdots
$$

in $\mathcal{F}_{C}$ such that $M \cong \operatorname{im}\left(W_{0} \rightarrow W^{0}\right)$ and $\mathbb{W} \bullet$ is $\operatorname{Hom}_{R}\left(\mathcal{P}_{C},-\right)$ and $\mathcal{I}_{C} \otimes_{R}$ exact.

It is clear that each module in $\mathcal{F}_{C}$ is $\mathcal{W}_{F}$-Gorenstein, and every kernel of $\mathbb{W}_{\bullet}$ is $\mathcal{W}_{F}$-Gorenstein.

In the following, we denote by $\mathcal{G}\left(\mathcal{W}_{F}\right)$ the class of $\mathcal{W}_{F}$-Gorenstein modules.

Proposition 3.2. $\mathcal{P}_{C} \perp \mathcal{G}\left(\mathcal{W}_{F}\right)$ and $\mathcal{I}_{C} \top \mathcal{G}\left(\mathcal{W}_{F}\right)$.

Proof. It follows directly from Lemmas 2.4 and 2.14.

Proposition 3.3. Let $\mathbb{W}_{\bullet}=\cdots \rightarrow W_{1} \rightarrow W_{0} \rightarrow W^{0} \rightarrow W^{1} \rightarrow \cdots$ be an exact sequence in $\mathcal{F}_{C}$ and $M \cong \operatorname{im}\left(W_{0} \rightarrow W^{0}\right)$. Then $\mathbb{W} \bullet$ is $\operatorname{Hom}_{R}\left(\mathcal{P}_{C},-\right)$ exact if and only if $M \in \mathcal{B}_{C}$.

Proof. Suppose $M \in \mathcal{B}_{C}$. By Lemma 2.13, every kernel of $\mathbb{W} \bullet$ is in $\mathcal{B}_{C}$, and so $\mathbb{W}_{\bullet}$ is $\operatorname{Hom}_{R}\left(P_{C},-\right)$ exact by Lemmas 2.4 and 2.14 .

Conversely, if $\mathbb{W}_{\bullet}$ is $\operatorname{Hom}_{R}\left(\mathcal{P}_{C},-\right)$ exact, then by Lemmas 2.4 and 2.14, we have $\mathcal{P}_{C} \perp M$. Hence, there exists an exact sequence

$$
\cdots \longrightarrow W_{1} \longrightarrow W_{0} \longrightarrow I^{0} \longrightarrow I^{1} \longrightarrow \cdots
$$

in $R$-Mod with each $I^{i}$ injective such that $M \cong \operatorname{im}\left(W_{0} \rightarrow I^{0}\right)$ and $\operatorname{Hom}_{R}\left(\mathcal{P}_{C},-\right)$ leaves it exact. Thus, $M \in \mathcal{B}_{C}$ by [7, Theorem 6.1].

Now we are in a position to give the result linking the classes $\mathcal{G F}_{C}$ and $\mathcal{G}\left(\mathcal{W}_{F}\right)$.

Theorem 3.4. Let $M$ be an $R$-module. Then $M \in \mathcal{G}\left(\mathcal{W}_{F}\right)$ if and only if $M \in \mathcal{G F}_{C} \cap \mathcal{B}_{C}$.

Proof. $(\Rightarrow)$. Let $M \in \mathcal{G}\left(\mathcal{W}_{F}\right)$. We first have $\mathcal{I}_{C} \top M$ by Proposition 3.2. Then $M \in \mathcal{G} \mathcal{F}_{C} \cap \mathcal{B}_{C}$ by Lemma 2.11 and Proposition 3.3. 
$(\Leftarrow)$. Let $M \in \mathcal{G} \mathcal{F}_{C} \cap \mathcal{B}_{C}$. Since $M \in \mathcal{G} \mathcal{F}_{C}$, we have that $\mathcal{I}_{C} \top M$, and there exists an exact sequence

$$
0 \longrightarrow M \longrightarrow W^{0} \longrightarrow W^{1} \longrightarrow \cdots
$$

in $R$-Mod with each $W^{i} \in \mathcal{F}_{C}$ such that $\mathcal{I}_{C} \otimes_{R}$-leaves it exact by Lemma 2.11. On the other hand, since $M \in \mathcal{B}_{C}$, it is easy to verify that $M$ has a proper left $\mathcal{P}_{C}$-resolution

$$
\cdots \longrightarrow V_{1} \longrightarrow V_{0} \longrightarrow M \longrightarrow 0 \text {. }
$$

It follows from Lemmas 2.13 and 2.14 that $\mathcal{I}_{C} \otimes_{R}-$ leaves it exact. Thus, we have the following exact sequence:

$$
\cdots \longrightarrow V_{1} \longrightarrow V_{0} \longrightarrow W^{0} \longrightarrow W^{1} \longrightarrow \cdots
$$

such that $M \cong \operatorname{im}\left(V_{0} \rightarrow W^{0}\right)$. By Proposition 3.3, we know that $\operatorname{Hom}_{R}\left(\mathcal{P}_{C},-\right)$ leaves it exact. It follows that $M \in \mathcal{G}\left(\mathcal{W}_{F}\right)$.

The following equivalence is comparable to [4, Theorem 3.11].

Theorem 3.5. There exists equivalence of categories:

$$
\mathcal{G}(\mathcal{F}) \cap \mathcal{A}_{C} \underset{\operatorname{Hom}_{R}(C,-)}{\gtrless} \mathcal{G}\left(\mathcal{W}_{F}\right) .
$$

Proof. We first show that the functor $\operatorname{Hom}_{R}(C,-)$ maps $\mathcal{G}\left(\mathcal{W}_{F}\right)$ to $\mathcal{G}(\mathcal{F}) \cap \mathcal{A}_{C}$. Assume $M \in \mathcal{G}\left(\mathcal{W}_{F}\right)$. Then there exists an exact sequence:

$$
\mathbb{W}_{\bullet}=\cdots \longrightarrow W_{1} \longrightarrow W_{0} \longrightarrow W^{0} \longrightarrow W^{1} \longrightarrow \cdots
$$

in $\mathcal{F}_{C}$ such that $M \cong \operatorname{im}\left(W_{0} \rightarrow W^{0}\right)$ and $\mathbb{W} \bullet$ is $\operatorname{Hom}_{R}\left(\mathcal{P}_{C},-\right)$ and $\mathcal{I}_{C} \otimes_{R^{-}}$exact. So $M \in \mathcal{B}_{C}$ by Theorem 3.4, and hence every kernel of $\mathbb{W}$ • is in $B_{C}$ by Lemma 2.13. Thus, $\operatorname{Hom}_{R}\left(C, \mathbb{W}_{\bullet}\right)$ is exact; moreover, $\operatorname{Hom}_{R}(C, M) \in \mathcal{A}_{C}$ by [7, Proposition 4.1]. On the other hand, suppose that $W_{i} \cong C \otimes_{R} F_{i}$ and $W^{i} \cong C \otimes_{R} F^{i}$, where each $F_{i}$ and $F^{i}$ flat. Then we have the following exact sequence in $R$-Mod:

$$
\operatorname{Hom}_{R}\left(C, \mathbb{W}_{\bullet}\right)=\cdots \longrightarrow F_{1} \longrightarrow F_{0} \longrightarrow F^{0} \longrightarrow F^{1} \longrightarrow \cdots
$$


such that $\operatorname{Hom}_{R}(C, M) \cong \operatorname{im}\left(F_{0} \rightarrow F^{0}\right)$. For each injective $R$-module $I$, we have

$$
\begin{aligned}
I \otimes_{R} \operatorname{Hom}_{R}(C, \mathbb{W} \bullet) & \cong C \otimes_{R} \operatorname{Hom}_{R}(C, I) \otimes_{R} \operatorname{Hom}_{R}\left(C, \mathbb{W}_{\bullet}\right) \\
& \cong \operatorname{Hom}_{R}(C, I) \otimes_{R} \mathbb{W} \bullet
\end{aligned}
$$

is exact. Hence, $\operatorname{Hom}_{R}(C, M) \in \mathcal{G}(\mathcal{F})$.

The proof of $C \otimes_{R}$-maps $\mathcal{G}(\mathcal{F}) \cap \mathcal{A}_{C}$ to $\mathcal{G}\left(\mathcal{W}_{F}\right)$ is similar.

The next result on the properties of the class $\mathcal{G}\left(\mathcal{W}_{F}\right)$ will be used frequently in the sequel.

Corollary 3.6. Let $R$ be a GF-closed ring. Then the class $\mathcal{G}\left(\mathcal{W}_{F}\right)$ is closed under extensions, kernels of epimorphisms and direct summands.

Proof. We first show that the class $\mathcal{G}\left(\mathcal{W}_{F}\right)$ is closed under extensions when $R$ is GF-closed. Consider the following short exact sequence:

$$
0 \longrightarrow M \longrightarrow N \longrightarrow K \longrightarrow 0
$$

with $M$ and $K$ belonging to $\mathcal{G}\left(\mathcal{W}_{F}\right)$. Since $M \in \mathcal{B}_{C}$ by Theorem 3.4, we get the next exact sequence:

$$
0 \longrightarrow \operatorname{Hom}_{R}(C, M) \longrightarrow \operatorname{Hom}_{R}(C, N) \longrightarrow \operatorname{Hom}_{R}(C, K) \longrightarrow 0 .
$$

It follows from Theorem 3.5 that $\operatorname{Hom}_{R}(C, M)$ and $\operatorname{Hom}_{R}(C, K)$ belong to $\mathcal{G}(\mathcal{F}) \cap \mathcal{A}_{C}$. Thus, $\operatorname{Hom}_{R}(C, N)$ belongs to $\mathcal{G}(\mathcal{F}) \cap \mathcal{A}_{C}$. On the other hand, since $N \in \mathcal{B}_{C}$ by Lemma 2.13 and Theorem 3.4, $N \cong C \otimes_{R} \operatorname{Hom}_{R}(C, N) \in \mathcal{G}\left(\mathcal{W}_{F}\right)$ by Theorem 3.5.

The proofs of the class $\mathcal{G}\left(\mathcal{W}_{F}\right)$ is closed under kernels of epimorphisms and direct summands are similar to [1, Theorem 2.3 and Corollary 2.6].

The next lemma will be used in the proof of Theorem 3.8.

Lemma 3.7. Let $R$ be a GF-closed ring. For every short exact sequence $0 \rightarrow G_{1} \rightarrow G_{0} \rightarrow M \rightarrow 0$ in $R$-Mod with $G_{0}, G_{1} \in \mathcal{G}\left(\mathcal{W}_{F}\right)$, if $\operatorname{Tor}_{1}^{R}\left(\mathcal{I}_{C}, M\right)=0$, then $M \in \mathcal{G}\left(\mathcal{W}_{F}\right)$. 
Proof. By the fact that the class $\mathcal{F}_{C}$ is a closed direct summand and [9, Lemma 4.1], the proof of the lemma is similar to [1, Theorem $2.3]$.

One can compare the following theorem on $\mathcal{G}\left(\mathcal{W}_{F}\right)$-projective dimension to [1, Theorem 2.8] and [8, Theorem 2.6].

Theorem 3.8. Let $R$ be a GF-closed ring and $M$ an $R$-module with finite $\mathcal{G}\left(\mathcal{W}_{F}\right)$-projective dimension. Let $n$ be a non-negative integer. Then the following are equivalent:

(i) $\mathcal{G}\left(\mathcal{W}_{F}\right)-\operatorname{pd}_{R}(M) \leqslant n$.

(ii) For every non-negative integer $t$ such that $0 \leqslant t \leqslant n$, there exists an exact sequence $0 \rightarrow W_{n} \rightarrow \cdots \rightarrow W_{1} \rightarrow W_{0} \rightarrow M \rightarrow 0$ in $R$-Mod such that $W_{t} \in \mathcal{G}\left(\mathcal{W}_{F}\right)$ and $W_{i} \in \mathcal{F}_{C}$ for $i \neq t$.

(iii) There exists a short exact sequence $0 \rightarrow K \rightarrow G \rightarrow M \rightarrow 0$ in $R$-Mod with $G \in \mathcal{G}\left(\mathcal{W}_{F}\right)$ and $\mathcal{F}_{C}-\operatorname{pd}_{R}(K) \leqslant n-1$.

(iv) There exists a short exact sequence $0 \rightarrow M \rightarrow K^{\prime} \rightarrow G^{\prime} \rightarrow 0$ in $R$-Mod with $G^{\prime} \in \mathcal{G}\left(\mathcal{W}_{F}\right)$ and $\mathcal{F}_{C}-\operatorname{pd}_{R}\left(K^{\prime}\right) \leqslant n$.

(v) There exists an exact sequence $0 \rightarrow G \rightarrow V_{n-1} \rightarrow \cdots \rightarrow V_{0} \rightarrow$ $M \rightarrow 0$ in $R$-Mod with $G \in \mathcal{G}\left(\mathcal{W}_{F}\right)$ and $V_{i} \in \mathcal{P}_{C}$ for all $0 \leqslant i \leqslant n-1$.

(vi) For every exact sequence $0 \rightarrow K_{n} \rightarrow G_{n-1} \rightarrow \cdots \rightarrow G_{0} \rightarrow M \rightarrow 0$ in $R$-Mod with $G_{i} \in \mathcal{G}\left(\mathcal{W}_{F}\right)$ for all $0 \leqslant i \leqslant n-1$, then also $K_{n} \in \mathcal{G}\left(\mathcal{W}_{F}\right)$.

(vii) $\operatorname{Tor}_{R}^{n+j}(U, M)=0$ for all $j \geqslant 1$ and all $U \in \mathcal{I}_{C}$.

(viii) $\operatorname{Tor}_{R}^{n+j}(U, M)=0$ for all $j \geqslant 1$ and all $U$ with $\mathcal{I}_{C}-r m i d_{R}(U)<$ $\infty$.

Furthermore, we have

$$
\begin{aligned}
& \mathcal{G}\left(\mathcal{W}_{F}\right)-\operatorname{pd}_{R}(M)=\sup \{n \in \mathbb{N} \mid\left.\operatorname{Tor}_{R}^{n}(U, M) \neq 0 \text { for some } U \in \mathcal{I}_{C}\right\} \\
&=\sup \left\{n \in \mathbb{N} \mid \operatorname{Tor}_{R}^{n}(U, M) \neq 0 \text { for some } U\right. \text { with } \\
&\left.\operatorname{I}_{C} \text {-id }{ }_{R}(U)<\infty\right\} .
\end{aligned}
$$

Proof. (ii) $\Rightarrow$ (iii) $\Rightarrow$ (i) and (vi) $\Rightarrow$ (i) are clear.

(i) $\Rightarrow$ (vii) $\Rightarrow$ (viii) follow from the usual dimension shifting argument. 
$(1) \Rightarrow$ (ii). Since the class $\mathcal{G}\left(\mathcal{W}_{F}\right)$ is closed under extensions by Corollary 3.6, the proof is similar to [8, Theorem 2.6].

(iii) $\Rightarrow$ (iv). Since $G \in \mathcal{G}\left(\mathcal{W}_{F}\right)$, there exists a short exact sequence $0 \rightarrow G \rightarrow W \rightarrow G^{\prime} \rightarrow 0$ in $R$-Mod with $W \in \mathcal{F}_{C}$ and $G^{\prime} \in \mathcal{G}\left(\mathcal{W}_{F}\right)$. Now consider the following push-out diagram:

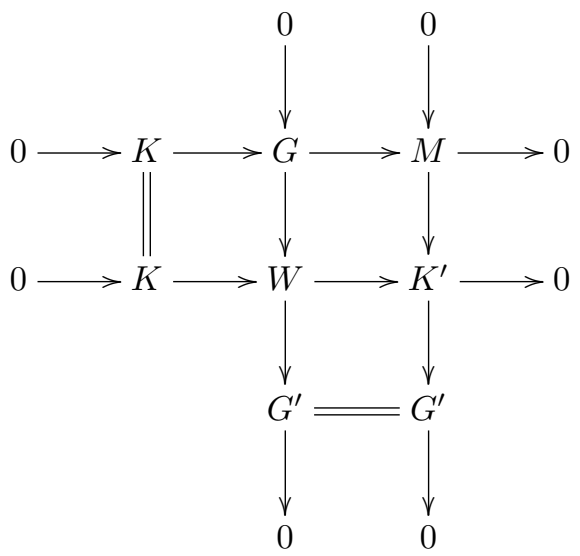

From the second row in the above diagram, we know $\mathcal{F}_{C}-\operatorname{pd}_{R}\left(K^{\prime}\right) \leqslant n$. So the third column is as desired.

(iv) $\Rightarrow$ (iii). Since $\mathcal{F}_{C}$-pd $\operatorname{pd}_{R}\left(K^{\prime}\right) \leqslant n$, there exists a short exact sequence $0 \rightarrow K \rightarrow W \rightarrow K^{\prime} \rightarrow 0$ in $R$-Mod with $W \in \mathcal{F}_{C}$ and $\mathcal{F}_{C}-\operatorname{pd}_{R}(K) \leqslant n-1$. Then consider the following pullback diagram: 


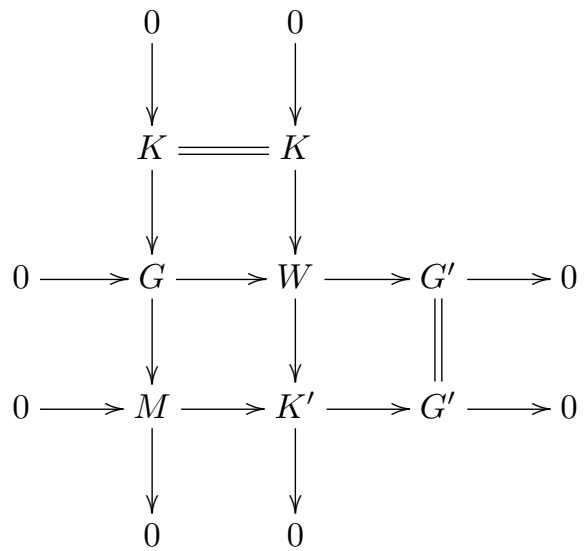

From the second row, we know that $G \in \mathcal{G}\left(\mathcal{W}_{F}\right)$ by Corollary 3.6. So the first column is as desired.

(i) $\Rightarrow(\mathrm{v})$. It suffices to prove the case $n=1$. Assume that $\mathcal{G}\left(\mathcal{W}_{F}\right)$ $\operatorname{pd}_{R}(M) \leqslant 1$. Then there exists a short exact sequence $0 \rightarrow G_{1} \rightarrow$ $G_{0} \rightarrow M \rightarrow 0$ in $R$-Mod with $G_{0}, G_{1} \in \mathcal{G}\left(\mathcal{W}_{F}\right)$. By Theorem 3.4, we know that $G_{0} \in \mathcal{B}_{C}$. Hence, it is easy to verify that there exists a short exact sequence $0 \rightarrow G_{0}^{\prime} \rightarrow V \rightarrow G_{0} \rightarrow 0$ in $R$-Mod such that $V \in \mathcal{P}_{C}$, and also $V \in \mathcal{G}\left(\mathcal{W}_{F}\right)$. By Corollary 3.6, we have $G_{0}^{\prime} \in \mathcal{G}\left(\mathcal{W}_{F}\right)$. Now consider the following pullback diagram:

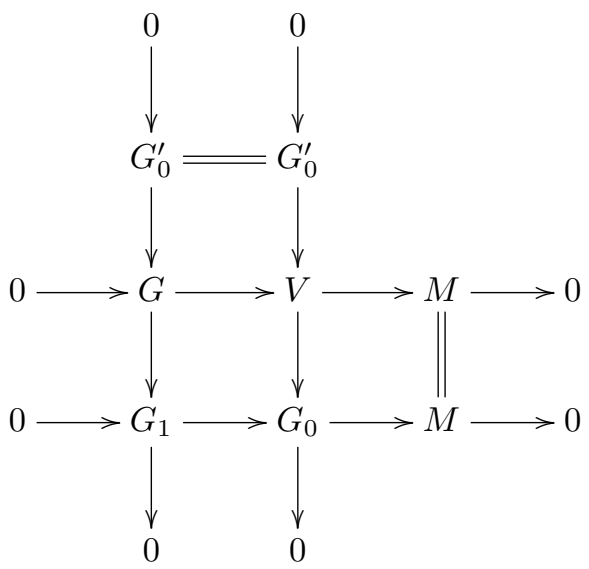


From the first column in the above diagram, we know that $G \in \mathcal{G}\left(\mathcal{W}_{F}\right)$ by Corollary 3.6. So the middle row is as desired.

(v) $\Rightarrow$ (vi). Let $0 \rightarrow K_{n} \rightarrow G_{n-1} \rightarrow \cdots \rightarrow G_{0} \rightarrow M \rightarrow 0$ be an exact sequence in $R$-Mod with each $G_{i} \in \mathcal{G}\left(\mathcal{W}_{F}\right)$. Then also $G_{i} \in \mathcal{B}_{C}$ by Theorem 3.4. Hence, $K_{n} \in \mathcal{B}_{C}$ and $\mathcal{P}_{C} \perp K_{n}$ by Lemmas 2.13 and 2.14. Then we have the following commutative diagram with exact rows:

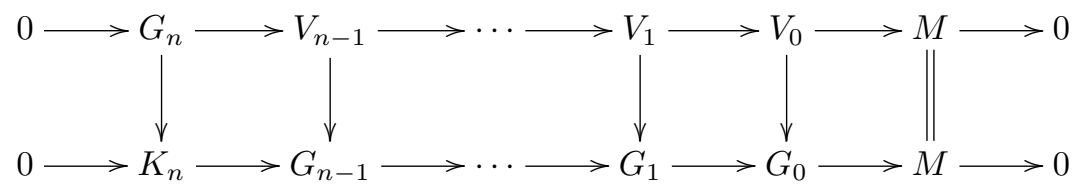

Thus, the mapping cone

$$
0 \longrightarrow G_{n} \longrightarrow V_{n-1} \oplus K_{n} \longrightarrow \cdots \longrightarrow V_{0} \oplus G_{1} \longrightarrow G_{0} \longrightarrow 0
$$

is exact. It follows from Corollary 3.6 that $K_{n} \in \mathcal{G}\left(\mathcal{W}_{F}\right)$.

(viii) $\Rightarrow$ (i). By Lemma 3.7, the proof is similar to [1, Theorem 2.8].

The last claim is an immediate consequence of the equivalence of (i), (vii) and (viii).

Let $n$ be a non-negative integer. In what follows, we denote by $\mathcal{G}$-flat $\leqslant n$ (respectively, $\mathcal{G}_{C}$-flat $\leqslant n$ ) the class of modules with finite Gorenstein flat (respectively, $\mathcal{G}\left(\mathcal{W}_{F}\right)$-projective) dimension at most $n$.

Theorem 3.9. (Foxby equivalence). Let $\mathcal{F}$ be the class of flat modules. There are equivalences of categories: 


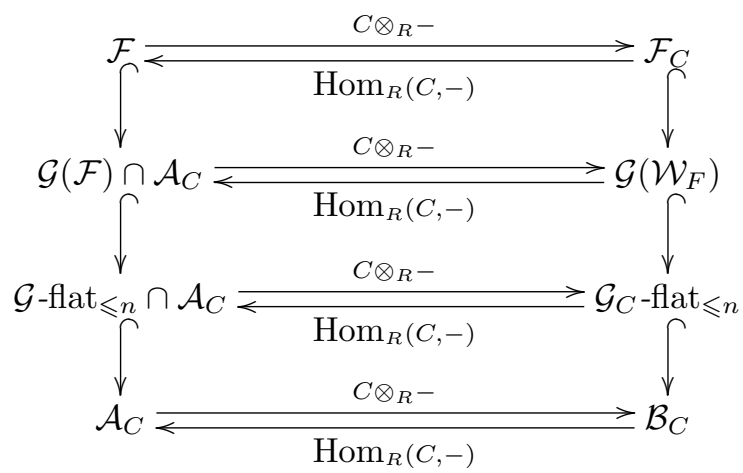

Proof. Let $M$ be an $R$-module. It suffices to prove the equivalence of categories of the third row in the above diagram.

For the third row, it suffices to prove the case $n=1$. Assume that $M \in \mathcal{G}_{C}$-flat $\leqslant 1$. Then there exists a short exact sequence

$$
0 \longrightarrow G_{1} \longrightarrow G_{0} \longrightarrow M \longrightarrow 0
$$

in $R$-Mod with $G_{0}, G_{1} \in \mathcal{G}\left(\mathcal{W}_{F}\right)$. Since $G_{1} \in \mathcal{B}_{C}$ by Theorem 3.4, we have the following exact sequence in $R$-Mod:

$$
0 \longrightarrow \operatorname{Hom}_{R}\left(C, G_{1}\right) \longrightarrow \operatorname{Hom}_{R}\left(C, G_{0}\right) \longrightarrow \operatorname{Hom}_{R}(C, M) \longrightarrow 0
$$

with $\operatorname{Hom}_{R}\left(C, G_{0}\right), \operatorname{Hom}_{R}\left(C, G_{1}\right) \in \mathcal{G}(\mathcal{F}) \cap \mathcal{A}_{C}$ by Theorem 3.5. Hence, by Lemma 2.13, $\operatorname{Hom}_{R}(C, M) \in \mathcal{G}$-flat $\leqslant 1 \cap \mathcal{A}_{C}$.

Conversely, assume that $M \in \mathcal{G}$-flat $\leqslant 1 \cap \mathcal{A}_{C}$. Then there exists a short exact sequence $0 \rightarrow G_{1} \rightarrow G_{0} \rightarrow M \rightarrow 0$ in $R$-Mod with $G_{0}, G_{1} \in \mathcal{G}(\mathcal{F}) \cap \mathcal{A}_{C}$. Since $M \in \mathcal{A}_{C}$ by Lemma 2.13 , Tor ${ }_{R}^{\geqslant 1}(C, M)=0$. Thus, there exists a short exact sequence:

$$
0 \longrightarrow C \otimes_{R} G_{1} \longrightarrow C \otimes_{R} G_{0} \longrightarrow C \otimes_{R} M \longrightarrow 0
$$

in $R$-Mod. By Theorem 3.5, we know that $C \otimes_{R} G_{0}, C \otimes_{R} G_{1} \in \mathcal{G}\left(\mathcal{W}_{F}\right)$. Hence, $C \otimes_{R} M \in \mathcal{G}_{C}$-flat $\leqslant 1$. 
4. Stability of categories. We start with the following definition.

Definition 4.1. Let $M$ be an $R$-module and $n \geqslant 2$ an integer. We say that $M \in \mathcal{G}^{n}\left(\mathcal{W}_{F}\right)$ if there exists an exact sequence

$$
\mathbb{G}_{\bullet}=\cdots \longrightarrow G_{1} \longrightarrow G_{0} \longrightarrow G^{0} \longrightarrow G^{1} \longrightarrow \cdots
$$

in $\mathcal{G}^{n-1}\left(\mathcal{W}_{F}\right)$ such that $M \cong \operatorname{im}\left(G_{0} \rightarrow G^{0}\right)$ and $\mathbb{G}_{\bullet}$ is

$$
\operatorname{Hom}_{R}\left(\mathcal{G}^{n-1}\left(\mathcal{W}_{F}\right),-\right)
$$

and

$$
\mathcal{G}^{n-1}\left(\mathcal{W}_{F}\right)^{+} \otimes_{R} \text {-exact. }
$$

Set $\mathcal{G}^{0}\left(\mathcal{W}_{F}\right)=\mathcal{F}_{C}, \mathcal{G}^{1}\left(\mathcal{W}_{F}\right)=\mathcal{G}\left(\mathcal{W}_{F}\right)$. One can easily check that there is a certain $\mathcal{G}^{n}\left(\mathcal{W}_{F}\right) \subseteq \mathcal{G}^{n+1}\left(\mathcal{W}_{F}\right)$ for all $n \geqslant 0$.

Similarly, one can also define modules which belong to $\mathcal{G}^{n}\left(\mathcal{G F}_{C} \cap \mathcal{B}_{C}\right)$ or $\mathcal{G}^{n}(\mathcal{F})$ for $n \geqslant 2$.

The next two results are given in service of the proof of Lemma 4.4.

Lemma 4.2. $\mathcal{P}_{C} \perp \mathcal{G}^{2}\left(\mathcal{W}_{F}\right)$ and $\mathcal{I}_{C} \top \mathcal{G}^{2}\left(\mathcal{W}_{F}\right)$.

Proof. It follows directly from Lemma 2.4, Proposition 3.2, and the fact that $\mathcal{P}_{C} \subseteq \mathcal{G}\left(\mathcal{W}_{F}\right)$ and $\mathcal{I}_{C} \subseteq \mathcal{G}\left(\mathcal{W}_{F}\right)^{+}$.

Lemma 4.3. Let $R$ be a GF-closed ring. Then $\mathcal{P}_{C}$ is a projective generator for $\mathcal{G}\left(\mathcal{W}_{F}\right)$.

Proof. Let $M$ be an $R$-module and $M \in \mathcal{G}\left(\mathcal{W}_{F}\right)$. So $M \in \mathcal{B}_{C}$ by Theorem 3.4. Hence, we have a short exact sequence

$$
0 \longrightarrow M^{\prime} \longrightarrow C \otimes_{R} P \longrightarrow M \longrightarrow 0
$$

in $R$-Mod with $P$ projective. By Corollary 3.6, we know that $M^{\prime} \in$ $\mathcal{G}\left(\mathcal{W}_{F}\right)$.

On the other hand, it follows from Proposition 3.2 that $\mathcal{P}_{C} \perp \mathcal{G}\left(\mathcal{W}_{F}\right)$. Thus, $\mathcal{P}_{C}$ is a projective generator for $\mathcal{G}\left(\mathcal{W}_{F}\right)$. 
Lemma 4.4. Let $R$ be a GF-closed ring, and let $M$ be an $R$-module which belongs to $\mathcal{G}^{2}\left(\mathcal{W}_{F}\right)$. Then $M$ admits a proper left $\mathcal{P}_{C}$-resolution.

Proof. It follows directly from the definition of modules which belong to $\mathcal{G}^{2}\left(\mathcal{W}_{F}\right)$, Lemma 4.2, Lemma 4.3 and [10, Lemma $\left.2.2(\mathrm{~b})\right]$.

Now we can give another main result in the paper.

Theorem 4.5. Let $R$ be a GF-closed ring. Then we have $\mathcal{G}^{n}\left(\mathcal{W}_{F}\right)=$ $\mathcal{G}\left(\mathcal{W}_{F}\right)$ for all $n \geqslant 1$.

Proof. It suffices to prove the case $n=2$. Let $M$ be an $R$-module and $M \in \mathcal{G}^{2}\left(\mathcal{W}_{F}\right)$. Following from Lemma 4.4, we have the exact sequence

$$
(\alpha)=\cdots \longrightarrow C \otimes_{R} P_{1} \longrightarrow C \otimes_{R} P_{0} \longrightarrow M \longrightarrow 0
$$

in $R$-Mod with each $P_{i}$ projective such that $\operatorname{Hom}_{R}\left(\mathcal{P}_{C},-\right)$ leaves it exact. By Lemma 2.4, Lemma 2.14 and Lemma 4.2, we know that $\mathcal{I}_{C} \otimes_{R}-$ leaves $(\alpha)$ exact as well.

On the other hand, since $M \in \mathcal{G}^{2}\left(\mathcal{W}_{F}\right)$, there exists a short exact sequence $0 \rightarrow M \rightarrow G \rightarrow M^{\prime} \rightarrow 0$ in $R$-Mod with $G \in \mathcal{G}\left(\mathcal{W}_{F}\right)$ and $M^{\prime} \in \mathcal{G}^{2}\left(\mathcal{W}_{F}\right)$. Since $G \in \mathcal{G}\left(\mathcal{W}_{F}\right)$, there exists a short exact sequence $0 \rightarrow G \rightarrow C \otimes_{R} F^{0} \rightarrow G^{\prime} \rightarrow 0$ in $R$-Mod with $F^{0}$ flat and $G^{\prime} \in \mathcal{G}\left(\mathcal{W}_{F}\right)$. Then we have the push-out diagram 


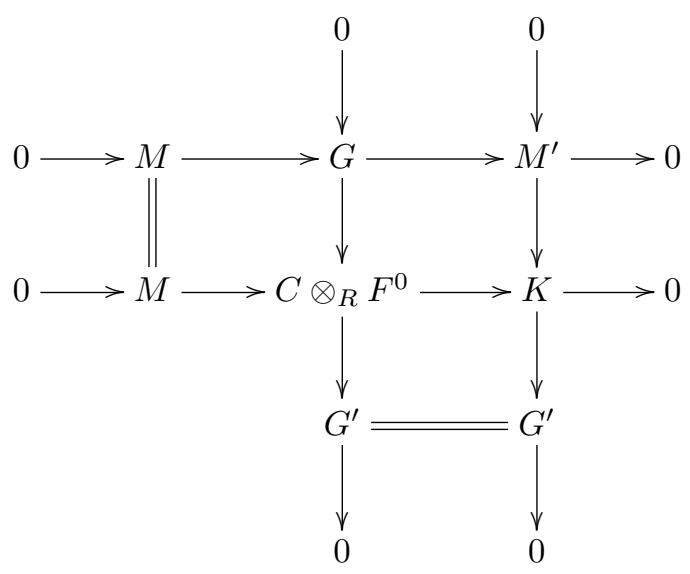

Consider the following short exact sequence coming from the middle row of the above diagram:

$$
(\beta)=0 \longrightarrow M \longrightarrow C \otimes_{R} F^{0} \longrightarrow K \longrightarrow 0 .
$$

From the third column of the above push-out diagram, we know that $\mathcal{I}_{C} \top K$ by Proposition 3.2 and Lemma 4.2. Hence, $(\beta)$ is $\operatorname{Hom}_{R}\left(\mathcal{P}_{C},-\right)$ and $\mathcal{I}_{C} \otimes_{R}$ - exact. If we can construct a short exact sequence

$$
(\eta)=0 \longrightarrow K \longrightarrow C \otimes_{R} F^{1} \longrightarrow K^{\prime} \longrightarrow 0
$$

in $R$-Mod with $F^{1}$ flat and $K^{\prime}$ a module with the same property as $K$ (that is, there exists a short exact sequence $(\mu)=0 \rightarrow M^{\prime \prime} \rightarrow K^{\prime} \rightarrow$ $H^{\prime \prime} \rightarrow 0$ in $R$-Mod with $M^{\prime \prime} \in \mathcal{G}^{2}\left(\mathcal{W}_{F}\right)$ and $\left.H^{\prime \prime} \in \mathcal{G}\left(\mathcal{W}_{F}\right)\right)$, then the following exact sequence can be constructed recursively:

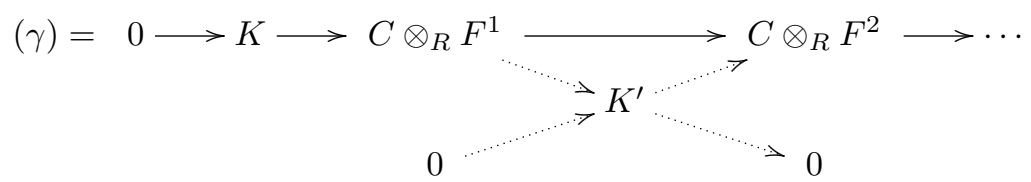

From the middle row of the above push-out diagram and $(\mu)$, we get $\mathcal{P}_{C} \perp K$ and $\mathcal{I}_{C} \top K^{\prime}$ by Proposition 3.2, Lemma 2.14 and Lemma 4.2. Then we have that $(\eta)$ is $\operatorname{Hom}_{R}\left(\mathcal{P}_{C},-\right)$ and $\mathcal{I}_{C} \otimes_{R}-$ exact. So is 
$(\gamma)$. Assembling the sequence $(\alpha),(\beta)$ and $(\gamma)$, we get the next exact sequence in $R$-Mod

$\cdots \longrightarrow C \otimes_{R} P_{1} \longrightarrow C \otimes_{R} P_{0} \longrightarrow C \otimes_{R} F^{0} \longrightarrow C \otimes_{R} F^{1} \longrightarrow \cdots$

such that $M \cong \operatorname{im}\left(C \otimes_{R} P_{0} \rightarrow C \otimes_{R} F^{0}\right)$, and $\operatorname{Hom}_{R}\left(\mathcal{P}_{C},-\right)$ and $\mathcal{I}_{C} \otimes_{R}$ - leave it exact. It follows that $M \in \mathcal{G}\left(\mathcal{W}_{F}\right)$.

Indeed, since $M^{\prime} \in \mathcal{G}^{2}\left(\mathcal{W}_{F}\right)$, there exists a short exact sequence $0 \rightarrow M^{\prime} \rightarrow H \rightarrow M^{\prime \prime} \rightarrow 0$ in $R$-Mod with $H \in \mathcal{G}\left(\mathcal{W}_{F}\right)$ and $M^{\prime \prime} \in \mathcal{G}^{2}\left(\mathcal{W}_{F}\right)$. Now consider the following push-out diagram:

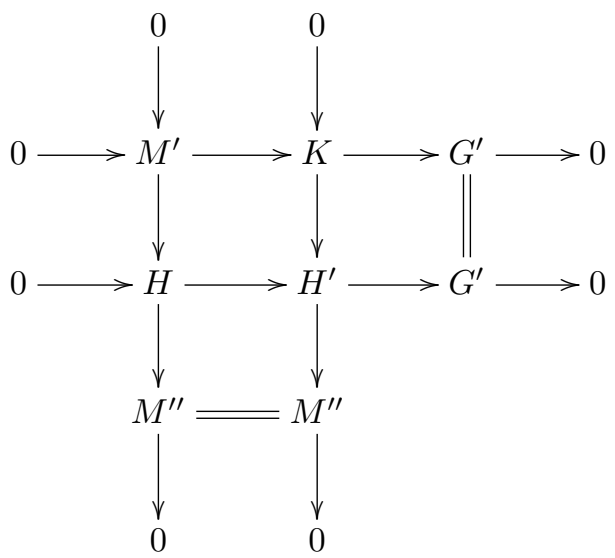

From the middle row of the above diagram, we know $H^{\prime} \in \mathcal{G}\left(\mathcal{W}_{F}\right)$ by Corollary 3.6. Then there exists a short exact sequence $0 \rightarrow H^{\prime} \rightarrow$ $C \otimes_{R} F \rightarrow H^{\prime \prime} \rightarrow 0$ in $R$-Mod with $F$ flat and $H^{\prime \prime} \in \mathcal{G}\left(\mathcal{W}_{F}\right)$. Consider another push-out diagram 


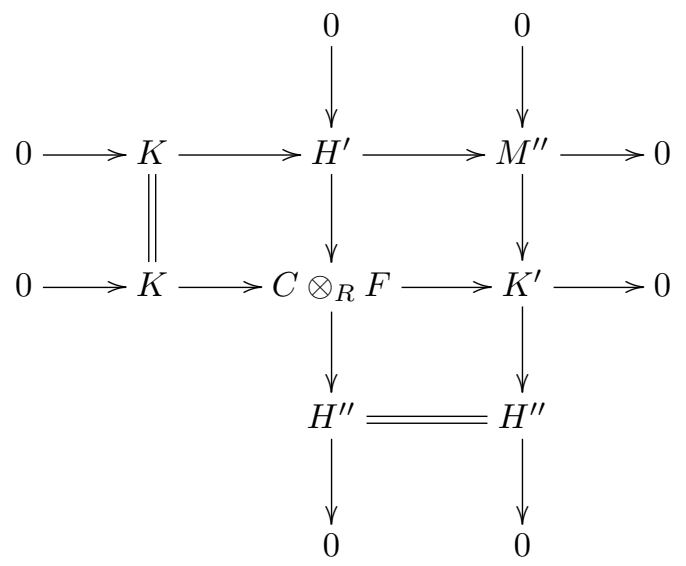

It is trivial that the third column in the above diagram is as desired. This completes the proof.

The following corollary is an immediate consequence of Theorem 3.4 and Theorem 4.5 .

Corollary 4.6. Let $R$ be a GF-closed ring. Then we have $\mathcal{G}^{n}\left(\mathcal{G F}_{C} \cap\right.$ $\left.\mathcal{B}_{C}\right)=\mathcal{G} \mathcal{F}_{C} \cap \mathcal{B}_{C}$ for all $n \geqslant 1$.

When we set $C=R$ in Corollary 4.6, we obtain the next result on the class of Gorenstein flat modules appeared in [12, Theorem 4.3] and $[2,1.2]$.

Corollary 4.7. Let $R$ be a GF-closed ring. Then we have $\mathcal{G}^{n}(\mathcal{F})=$ $\mathcal{G}(\mathcal{F})$ for all $n \geqslant 1$.

Acknowledgments. The authors are grateful for the helpful remarks of the referee.

\section{REFERENCES}

1. D. Bennis, Rings over which the class of Gorenstein flat modules is closed under extensions, Comm. Algebra 37 (2009), 855-868.

2. S. Bouchiba and M. Khaloui, Stability of Gorenstein flat modules, Glasgow Math. J. 54 (2012), 169-175. 
3. E.E. Enochs, O.M.G. Jenda and B. Torrecillas, Gorenstein flat modules, Nanjing Daxue Xuebao Shuxue Bannian Kan. 10 (1993), 1-9.

4. Y.X. Geng and N.Q. Ding, $\mathcal{W}$-Gorenstein modules, J. Algebra 325 (2011), $132-146$.

5. H. Holm, Gorenstein homological dimensions, J. Pure Appl. Alg. 189 (2004), $167-193$.

6. H. Holm and P. Jøgensen, Semi-dualizing modules and related Gorenstein homological dimensions, J. Pure Appl. Alg. 205 (2006), 423-445.

7. H. Holm and D. White, Foxby equivalence over associative rings, J. Math. Kyoto Univ. 47 (2007), 781-808.

8. C.H. Huang and Z.Y. Huang, Gorenstein syzygy modules, J. Alg. 324 (2010), 3408-3419.

9. S.S. Wagstaff, T. Sharif and D. White, AB-contexts and stability for Gorenstein flat modules with respect to semidualizing modules, Alg. Rep. Theor. 14 (2011), 403-428.

10. Stability of Gorenstein categories, J. Lond. Math. Soc. 77 (2008), $481-502$.

11. D. White, Gorenstein projective dimension with respect to a semidualizing module, J. Comm. Alg. 2 (2010), 111-137.

12. G. Yang and Z.K. Liu, Stability of Gorenstein flat category, Glasgow Math. J. 54 (2012), 177-191.

Department of Mathematics, Northwest Normal University, Lanzhou 730070 CHINA

Email address: dizhenxing19841111@126.com

Department of Mathematics, Northwest Normal University, Lanzhou 730070 CHINA

Email address: liuzk@nwnu.edu.cn

Department of Mathematics, Southeast University, Nanjing 210096 China

Email address: jlchen@seu.edu.cn 